\title{
Estudo cinético do desenvolvimento da microalga Chlorella Vulgaris em efluentes de tratamento anaeróbio de esgoto doméstico para fins de produção de biodiesel
}

Dentre as inúmeras possibilidades que visam à diminuição do uso de combustíveis fósseis, têm se destacado as microalgas, cuja biomassa pode ser utilizada na geração de diferentes formas de energia, além de sua elevada capacidade para a depuração de águas residuárias, absorção de $\mathrm{CO} 2$ e eficiência superior na produção de óleo em relação às oleaginosas. Este estudo teve como objetivo avaliar as melhores condições de crescimento da microalga Chlorella vulgaris em meios de cultura suplementados com efluente de tratamento anaeróbio de esgoto doméstico do reator tipo UASB, buscando reduzir o custo dos cultivos para a produção de biodiesel, bem como promover um destino adequado a esse efluente, impróprio para lançamento em corpos hídricos. A microalga em estudo foi cultivada utilizando o meio de cultura sintético BBM adicionado a diferentes proporções do efluente de UASB (20,30,50, 70, 80 e 100\%). De acordo com os parâmetros cinéticos de crescimento, observou-se que o efluente de UASB ofereceu condições para o ótimo desenvolvimento da microalga, resultando em acúmulo de biomassa, além de reduzir a carga poluidora do efluente. Concluiu-se que a utilização de resíduos líquidos oriundos do tratamento anaeróbio de esgoto doméstico pode aumentar a viabilidade dos cultivos visando à produção de biodiesel, uma vez que permitem redução de custos ao substituir de forma total ou parcial o meio sintético.

Palavras-chave: Microalgas; Chlorella vulgaris; Efluente de UASB; Biodiesel; Reuso.

\section{Kinetic study of the development of Chlorella Vulgaris microalgae in anaerobic domestic sewage treatment effluents for biodiesel production purposes} capacity for wastewater purification, $\mathrm{CO} 2$ absorption and efficiency. higher in oil production than in oilseeds. The objective of this study was to evaluate the best growth conditions of Chlorell vulgaris microalgae in culture media supplemented with anaerobic sewage treatment effluent from the UASB reactor, aiming to reduce the cost of crops for biodiesel production, as well as to promote a destination. suitable for this effluent, unsuitable for release into water bodies. The microalgae under study was cultivated using BBM synthetic culture medium added at differen proportions of UASB effluent (20,30,50,70,80 and 100\%). According to the kinetic parameters of growth, it was observed that UASB effluent provided conditions for the optimal development of microalgae, resulting in biomass accumulation, besides reducing the effluent pollutant load. It was concluded that the use of liquid waste from anaerobic treatment of domestic sewage can increase the viability of crops aiming at the production of biodiesel, since they allow cost reduction by totally or partially replacing the synthetic medium.

Keywords: Microalgae; Chlorella vulgaris; UASB Effluent; Biodiesel; Reuse.

Topic: Engenharia Sanitária

Reviewed anonymously in the process of blind peer.
Received: 04/10/2018

Approved: 04/11/2018
Thiago Santos de Almeida Lopes (id Universidade Federal da Paraíba, Brasil http://orcid.org/0000-0002-0270-6133

thiago.s.16@hotmail.com

Weruska Brasileiro Ferreira

Universidade Federal da Paraíba, Brasil weruska brasileiro@yahoo.com.br

Thyago Nóbrega Silveira

Universidade Federal de Campina Grande, Brasil http://lattes.cnpq.br/2919903964357018

thyagonobrega1996@gmail.com http://lattes.cnpq.br/6519791629716421 http://lattes.cnpq.br/5630172788119332

Célia Regina Diniz
Universidade Estadual da Paraíba, Brasil
http://lattes.cnpq.br/5852965121074357
$\underline{\text { c.r.diniz13@gmail.com }}$
Whelton Brito dos Santos (D)
Universidade Federal de Campina Grande, Brasil
http://lattes.cnpq.br/6407528093074339
$\underline{\text { http://orcid.org/0000-0002-2956-8260 }}$
wheltonbrt@gmail.com
Amanda Laurentino Torquato
Universidade Estadual da Paraíba, Brasil
http://lattes.cnpq.br/9586107560324398
amanda.torquato02@gmail.com

Líbia de Sousa Conrado Oliveira Universidade Federal de Campina Grande, Brasil

http://lattes.cnpq.br/1704346203363785 libiaconrado@yahoo.com.br
LOPES, T. S. A.; FERREIRA, W. B.; SILVEIRA, T. N.; DINIZ, C. R.; SANTOS, W. B.; TORQUATO, A. L.; OLIVEIRA, L. S. O.. Estudo cinético do desenvolvimento da microalga Chlorella Vulgaris em efluentes de tratamento anaeróbio de esgoto doméstico para fins de produção de biodiesel. Revista Ibero-Americana de Ciências Ambientais, v.9, n.8, p.70-81, 2018. DOI: http://doi.org/10.6008/CBPC2179$\underline{6858.2018 .008 .0007}$

\section{Referencing this:}

DOI: 10.6008/CBPC2179-6858.2018.008.0007 


\section{INTRODUÇÃO}

A crescente demanda por energia, associada à intensa utilização dos recursos naturais, tem sido responsável pelo estabelecimento de uma crise energética mundial. Combustíveis fósseis como o petróleo, o carvão mineral e o gás natural não são renováveis e estão fadados ao esgotamento. Além disso, promovem a emissão de gases poluentes como o dióxido de carbono, óxidos de nitrogênio e hidrocarbonetos, que provocam desequilíbrios ambientais e alterações climáticas (FERREIRA et al., 2013). Uma alternativa que tem ganhado relevância é o uso de biocombustíveis, onde o biodiesel se destaca como uma das opções mais viáveis para promover a gradativa redução do consumo de combustíveis fósseis, já que possibilita a substituição destes sem a necessidade de alteração de infraestruturas (FRANCO et al., 2013).

O biodiesel geralmente é produzido a partir de diferentes espécies de oleaginosas, como soja, mamona, canola, girassol e algodão. No entanto, a necessidade de grandes áreas para cultivo torna a produção em larga escala uma realidade distante, pois afeta diretamente a produção de alimentos, além de contribuir para a destruição da flora natural, esgotamento da capacidade dos solos e erradicação de espécies. $\mathrm{O}$ alto consumo de fertilizantes, pesticidas e água para irrigação também acarretam em problemas ambientais (CHIRANJEEVI et al., 2016).

As microalgas surgem como alternativa promissora para solução dessa questão, pois apresentam produtividade de lipídios superior à das culturas vegetais convencionalmente utilizadas e podem ser cultivadas em terras não agricultáveis, já que sua produção é não sazonal, possibilitando diversas colheitas ao longo de todo o ano. Além disso, possuem a capacidade de absorver grande quantidade de dióxido de carbono da atmosfera e de fontes poluentes (FERREIRA et al., 2013; MENEZES et al., 2013).

O teor de lipídios na biomassa depende diretamente da espécie de microalga selecionada para o cultivo e das condições ambientais a que este é submetido. As microalgas de espécie Chlorella vulgaris apresentam características fundamentais para a produção do biodiesel: são verdes, simples, eucarióticas, esféricas, apresentam elevada eficiência fotossintética, excelentes produtividades de biomassa e de lipídios e a sua estrutura celular simplifica o processamento do biodiesel quando comparado com outras plantas complexas (CHISTI, 2008; DANTAS et al., 2010).

Além disso, os ácidos graxos da Chlorella vulgaris apresentam o perfil adequado para a produção de biodiesel, com propriedades que cumprem os padrões dos EUA (ASTM D6751), da Europa (EM 14214) e da Agência Nacional de Petróleo do Brasil (ANP 255). Sua biomassa possui também alto teor de amido, que pode ser empregado na geração de bioetanol (SAFI et al., 2014). Contudo, apesar do potencial desta microalga, os meios de cultura sintéticos utilizados para seu crescimento apresentam custos elevados, tornando os cultivos em larga escala inviáveis do ponto de vista econômico. Como alternativa, tem sido estudada a substituição destes meios por águas residuárias (VIEIRA et al., 2014; MATOSI et al., 2015).

Atualmente, o reator UASB (Upflow Anaerobic Sludge Blanket) tem se consolidado como uma tendência em tratamento de esgotos no mundo, principalmente em regiões de clima tropical como Brasil e Índia (BRESSANI-RIBEIRO et al., 2017). Esse sistema apresenta vantagens, no que diz respeito à sua 
simplicidade operacional, quantidade de área requerida e baixo custo de manutenção e operação (CHERNICHARO, 2007). Contudo, sua limitada remoção de nutrientes reforça a necessidade de póstratamento do seu efluente.

Desse modo, quando ocorre a utilização de esgoto tratado em reator UASB como substrato para cultivo das microalgas, os custos com tecnologias de pós-tratamento são reduzidos, bem como os impactos ambientais advindos do lançamento desse efluente em corpos d'água receptores. Além disso, a substituição parcial ou total do meio de cultura sintético por esgoto tratado promove a redução dos custos dos cultivos e da demanda por água potável, aumentando a viabilidade econômica deste processo e possibilitando geração de energia renovável e sustentável. Nesse sentido, este estudo teve como objetivo avaliar o potencial biotecnológico da microalga Chlorella vulgaris para fins de produção de biodiesel, cultivada em efluente de reator UASB visando reduzir os custos dos cultivos e promover a destinação adequada desse resíduo líquido.

\section{METODOLOGIA}

\section{Microalga Chlorella vulgaris}

O estudo foi realizado no Laboratório de Saneamento Ambiental, localizado no Centro de Ciências e Tecnologia da Universidade Estadual da Paraíba, Campina Grande (PB), onde foram utilizadas cepas da microalga Chlorella vulgaris fornecidas pelo Laboratório de Biotecnologia Alimentar da Universidade Federal de Santa Catarina. A espécie em estudo (figura 1) foi escolhida devido à sua predominância nos meios aquáticos da região semiárida do Nordeste brasileiro, onde foram realizados os experimentos, bem como a sua elevada produtividade de lipídios e resistência a diferentes condições ambientais, o que favorece a produção de biodiesel. Apresenta-se como candidata em potencial para mitigação das emissões de dióxido de carbono e remediação de águas residuárias.

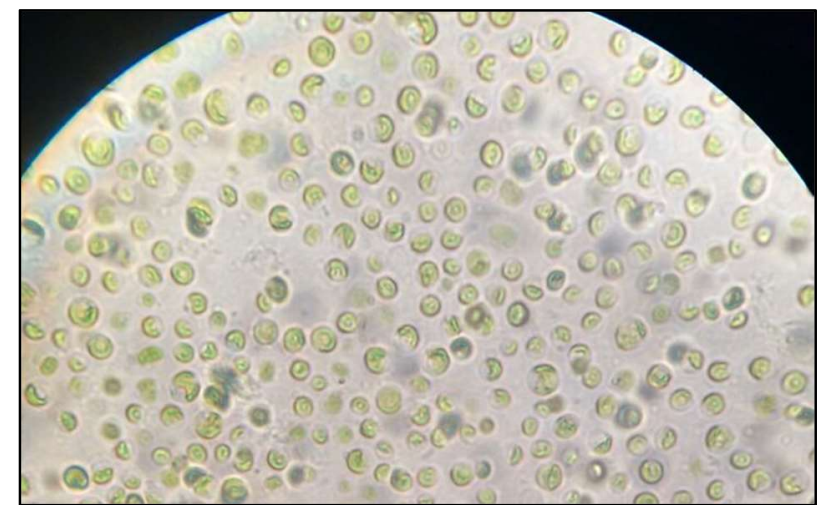

Figura 1: Fotomicrografia da microalga Chlorella vulgaris.

\section{Meio de cultivo}

Para o cultivo da Chlorella vulgaris foi utilizado o meio sintético Bold's Basal Medium (BBM), recomendado pelo Centro de Cultura de Algas e Protozoários de Cambridge, suplementado com extrato de levedura e efluente de reator UASB. O efluente de UASB apresentou DQO de $251 \mathrm{mg} . \mathrm{L}^{-1}$, após filtração para retenção de sólidos suspensos que poderiam interferir nos cultivos, e foi fornecido pela Estação Experimental 
de Tratamento Biológico de Esgotos Sanitários (EXTRABES), localizada na cidade de Campina Grande (PB). Com o intuito de verificar a concentração de melhor adaptação da microalga, o efluente de reator UASB foi adicionado em diferentes proporções (20\%, 30\%, 50\%, $70 \%, 80 \%$ e $100 \%)$ de volume do meio de cultura.

\section{Aclimatação dos cultivos}

A aclimatação dos cultivos, conforme se vê na figura 2, foi realizada de modo a estabelecer um ambiente propício para a otimização do crescimento da Chlorella vulgaris. Cada unidade experimental (fotobiorreator) consistiu-se em uma cultura microalgal desenvolvida em Erlenmeyer com volume de 500mL. Os fotobiorreatores foram mantidos a temperatura de $26 \pm 2^{\circ} \mathrm{C}$, com agitação de ar comprimido para garantir a homogeneização do meio, e expostos, por um período de 12 horas, a iluminação de quatro lâmpadas fluorescentes de 40 watts e 2.700 lumens cada.

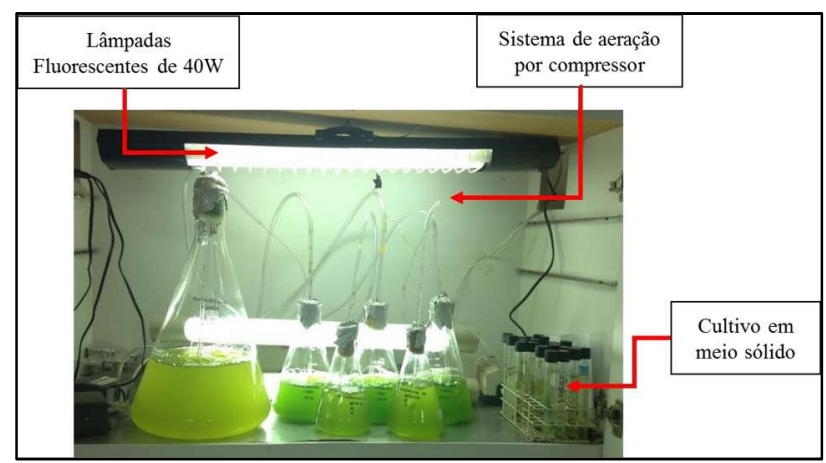

Figura 2: Aclimatação dos cultivos das microalgas.

\section{Avaliação do crescimento}

Com o auxílio da microscopia óptica (aumento de 400x) foi possível determinar o crescimento das microalgas avaliando-se a concentração celular em função do tempo de cultivo em cada uma das unidades experimentais. A contagem de células foi realizada em triplicata, através de uma câmara de Neubauer, onde o número de células, em células. $\mathrm{mL}^{-1}$, foi determinado pela média geométrica das três contagens. $O$ tempo de cultivo foi expresso pela quantidade de dias decorridos desde o início da inoculação (período de adaptação - fase lag) até o alcance máximo da concentração celular (fase estacionária). Cada experimento teve duração de 9 dias, dentre os quais foram retiradas 7 amostras para realização das contagens. Gráficos de dispersão foram plotados para representar as curvas de crescimento da Chlorella vulgaris, onde o eixo das abscissas corresponde ao tempo de cultivo em dias, e o eixo das ordenadas ao número de células. $\mathrm{mL}^{-1}$.

\section{Cálculo da velocidade específica de crescimento e tempo de geração}

A velocidade de crescimento é diretamente proporcional à concentração de microrganismos em um dado instante. A fração pela qual a população cresce na unidade de tempo é dada por $\mu_{x}$, que representa a velocidade específica de crescimento e é expressa em $\mathrm{h}^{-1}$. Na fase exponencial (ou logarítmica) a velocidade específica de crescimento é constante e máxima $\left(\mu_{\mathrm{x}}=\mu_{\text {máx }}\right)$ e pode ser determinada através da equação (SCHMIDELL et al., 2001). Tem-se, conforme proposto, $\ln X=\mu_{\text {máx }} \cdot\left(t-t_{i}\right)+\ln X_{i}$. 
A representação de $\operatorname{InX}$ versus o tempo de cultivo, na fase exponencial, resulta em uma reta com coeficiente angular igual à velocidade específica máxima de crescimento $\mu_{\text {máx. }}$. A fase exponencial também é caracterizada pelo tempo de geração $\left(\mathrm{t}_{\mathrm{g}}\right)$, que é o tempo necessário para dobrar o valor da concentração celular $\left(X=2 X_{i}\right)$. $O$ tempo de geração pode ser calculado através da equação seguinte (SCHMIDELL et al., 2001). Como foi proposto, tem-se $t_{g}=\frac{\ln 2}{\mu_{\text {máx }}}=\frac{0,693}{\mu_{\text {máx }}}$.

\section{Determinação da produtividade de biomassa}

A produtividade de biomassa foi determinada pela diferença entre os pesos da biomassa seca no início e no fim de cada cultivo, dividida pelo tempo total do experimento (9 dias). A biomassa de Chlorella vulgaris foi separada do meio de cultura por centrifugação, a uma rotação de 3500rpm, e colocada em cápsula de porcelana para secagem em estufa, na temperatura de $60^{\circ} \mathrm{C}$, até atingir peso constante para posterior pesagem. As análises foram realizadas em triplicata.

\section{Análise de remoção da Demanda Química de Oxigênio (DQO)}

Nos cultivos suplementados com efluente de reator UASB foram realizadas análises de DQO para verificar se a microalga Chlorella vulgaris promoveria remoção de matéria orgânica, implicando no tratamento do efluente. As análises foram executadas em triplicata, conforme método colorimétrico descrito no Standard Methods (RICE et al., 2012). Foram analisadas amostras de meio de cultura antes do inóculo das microalgas e ao término dos experimentos (após 9 dias). Nas amostras coletadas ao fim dos cultivos, as microalgas foram separadas do meio de cultura por centrifugação a 3500rpm, analisando-se os sobrenadantes. Desse modo, foi possível comparar os valores de DQO iniciais e finais e verificar se a Chlorella vulgaris realizou biorremediação no resíduo líquido em estudo.

\section{Extração de lipídios totais}

A extração de lipídios totais foi realizada a partir da biomassa seca, por meio da metodologia gravimétrica descrita por Bligh et al. (1959), utilizando-se metanol e clorofórmio como solventes em uma proporção de 2:1 (v/v). Devido à resistência da parede celular das microalgas, esta metodologia foi adaptada com a introdução de uma etapa de rompimento celular, onde as amostras foram submetidas a banho ultrassônico com frequência de $25 \mathrm{kHz}$, durante 20 minutos.

\section{Análise estatística}

Foi efetuado o estudo da matriz de correlação com o objetivo de se verificar o nível de relação entre as variáveis analisadas. Além deste, executou-se também um estudo estatístico multivariado dos dados por meio da Análise em Componentes Principais (ACP), cujo intuito foi se estudar a formação dos grupos de variabilidades equivalentes. $O$ critério adotado para seleção de variáveis foi a existência de uma correlação 
mínima de +/- 0,6 entre a coluna da variável e pelo menos duas outras variáveis. Para estas análises foram utilizados os softwares Microsoft Excel 2016 e Statistic 7.0.

\section{RESULTADOS E DISCUSSÃO}

\section{Curvas de crescimento da Chlorella vulgaris}

Para se avaliar o crescimento da Chlorella vulgaris em função das diferentes concentrações de efluente de reator UASB adicionadas aos cultivos, foram plotadas curvas de crescimento para cada experimento, em um período de cultivo de 9 dias (figura 3). A partir destas, pôde-se observar que todas as concentrações do efluente promoveram um excelente desenvolvimento celular das microalgas, resultando em concentrações de células na ordem de $10^{7}$ células. $\mathrm{mL}^{-1}$ ao final dos experimentos.

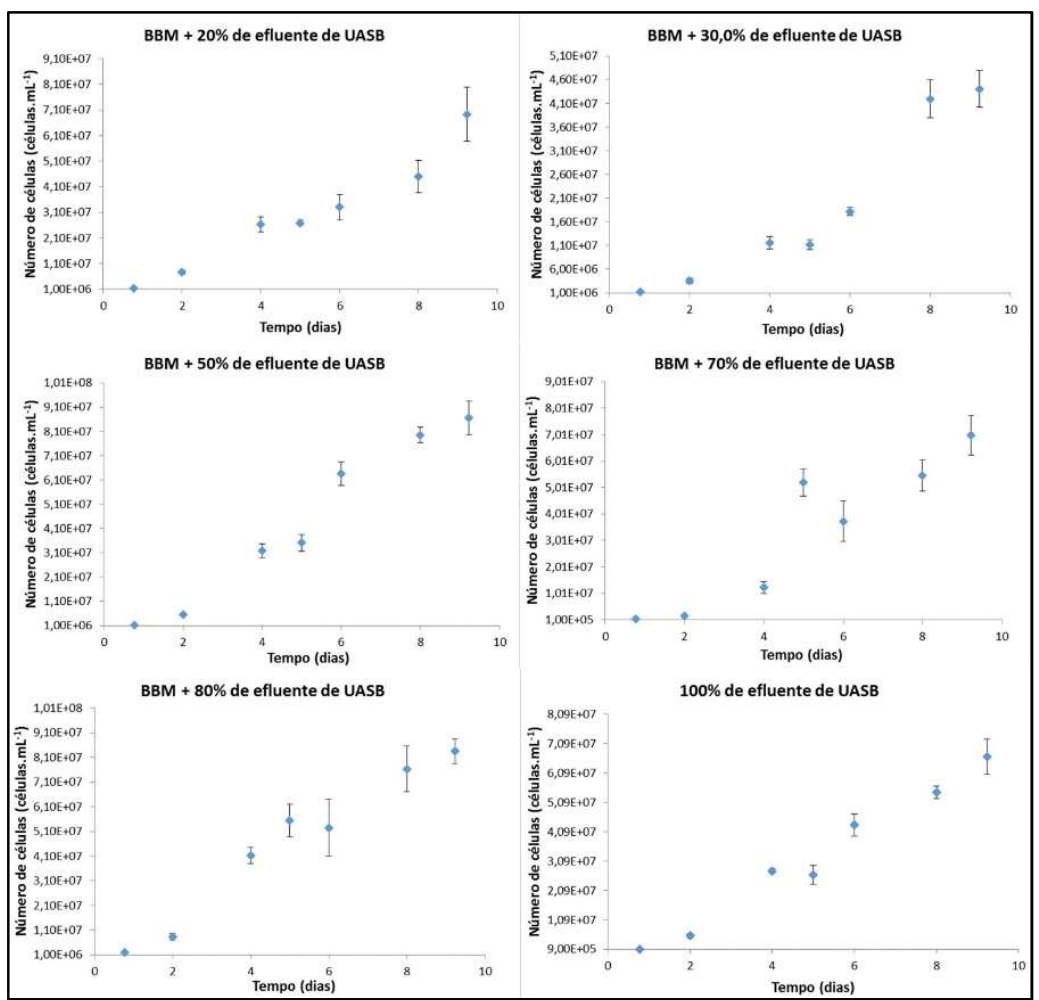

Figura 3: Curvas de crescimento da Chlorella vulgaris em meio BBM suplementado com diferentes proporções de efluente de UASB.

Os dados experimentais revelam que em meio de cultivo BBM contendo $20 \%$ de efluente de UASB as microalgas apresentam um elevado crescimento, que iniciou com $1,36 \times 10^{6}$ células. $\mathrm{mL}^{-1}$ e terminou com $6,93 \times 10^{7}$ células. $\mathrm{mL}^{-1}$. A curva de crescimento em meio de cultivo BBM contendo $30 \%$ de efluente de UASB também evidencia ótimo crescimento. 0 cultivo iniciou com $1,26 \times 10^{6}$ células. $\mathrm{mL}^{-1}$ e foi finalizado com $4,41 \times 10^{7}$ células $\mathrm{mL}^{-1}$.

Dantas et al. (2010) estudou o crescimento da Chlorella vulgaris em meio de cultura Conway modificado, com fotoperíodo de 12 horas em fotobioreatores de vidro de $250 \mathrm{~mL}$ com intensidade luminosa de 4000 lux, obtendo uma densidade máxima celular no oitavo dia de cultivo de $2,5 \times 10^{7}$ células. $\mathrm{mL}^{-1}$. Junior et al. (2013) analisou o crescimento da mesma microalga em efluentes suínos, onde a concentração de 
melhor adaptação, efluente diluído a 1\%, permitiu que as microalgas chegassem a uma concentração máxima de $5,37 \times 10^{6}$ células. $\mathrm{mL}^{-1}$, ao sexto dia de cultivo.

Vieira et al. (2014) avaliou o crescimento da Chlorella sp. em meio de cultura BBM suplementado com glicerina e vinhaça, obtendo como melhores resultados as concentrações máximas de $2,7 \times 10^{6}$ células. $\mathrm{mL}^{-1}$ em cultivo com $2 \%$ de glicerina, e $2,57 \times 10^{7}$ células. $\mathrm{mL}^{-1}$ em cultivo com $10 \%$ de vinhaça. Isso mostra que, apesar do cultivo da microalga Chlorella vulgaris com 30\% de efluente de UASB ter desenvolvido um crescimento inferior ao do cultivo com $20 \%$, ainda assim apresentou um crescimento satisfatório quando comparado com os estudos supracitados.

Os dados experimentais da Chlorella vulgaris em meio de cultivo BBM contendo 50\% de efluente de UASB apontam que ele foi o que desenvolveu o maior número de células no tempo de 9 dias. Iniciou com $1,06 \times 10^{6}$ células. $\mathrm{mL}^{-1}$ e finalizou com $8,66 \times 10^{7}$ células. $\mathrm{mL}^{-1}$, demostrando que os nutrientes presentes no efluente de UASB favoreceram o crescimento das microalgas.

O cultivo de Chlorella vulgaris em meio de cultivo BBM contendo 70\% de efluente de UASB iniciou com $2,61 \times 10^{5}$ células. $\mathrm{mL}^{-1}$ e, após 9 dias, com $6,98 \times 10^{7}$ células. $\mathrm{mL}^{-1}$. Apresentou excelente crescimento celular, visto que começou com uma concentração inferior de células (na ordem de $10^{5}$ ) quando comparado com os demais cultivos (que iniciaram na ordem de $10^{6}$ ). A curva de crescimento da Chlorella vulgaris em BBM com $80 \%$ de efluente de UASB confirma sua não toxicidade para as microalgas, uma vez que, mesmo em altas concentrações no meio de cultura, possibilita o crescimento das mesmas. O cultivo iniciou com $1,67 \times 10^{6}$ células. $\mathrm{mL}^{-1}$ e finalizou com $2,26 \times 10^{7}$ células. $\mathrm{mL}^{-1}$.

Assim como o cultivo anterior, os dados experimentais da Chlorella vulgaris cultivada em $100 \%$ de efluente de UASB, sem o meio sintético BBM, exprimiram excelente e constante crescimento das microalgas, mostrando que apenas os nutrientes presentes no efluente de UASB são suficientes para o seu desenvolvimento. $O$ cultivo iniciou com $9,23 \times 10^{5}$ células. $\mathrm{mL}^{-1}$, terminando com $6,66 \times 10^{7}$ células. $\mathrm{mL}^{-1}$.

Liang et al. (2009) estudaram o crescimento da Chlorella vulgaris em cultivo autotrófico (luz como única fonte de energia) e heterotrófico (somente compostos orgânicos como fonte energética) e observaram que a respectiva microalga apresentou bom desenvolvimento nas duas condições, sendo classificada como mixotrófica. Isso explica a adequação desta espécie às diferentes concentrações de efluente de UASB.

As microalgas, em sua diversidade, são organismos muito flexíveis quanto ao habitat, sendo encontradas tanto em ambientes úmidos terrestres, quanto em ambientes aquáticos de água doce, salobra e salgada. Esta capacidade proporciona vantagens consideráveis em comparação com os cultivos convencionais utilizados para a produção de biodiesel, com potencial de minimizar o uso e os impactos ambientais adversos nos recursos naturais solo e água (FRANCO et al., 2013). Isso pode ser observado com a adaptação da Chlorella vulgaris em efluentes do reator tipo UASB na ausência de meio sintético, o que pode aumentar a viabilidade do seu cultivo para a produção de biodiesel. 
Estudo cinético do desenvolvimento da microalga Chlorella Vulgaris em efluentes de tratamento anaeróbio de esgoto doméstico para fins de produção de biodiesel

LOPES, T. S. A.; FERREIRA, W. B.; SILVEIRA, T. N.; DINIZ, C. R.; SANTOS, W. B.; TORQUATO, A. L.; OLIVEIRA, L. S. O.

\section{Parâmetros cinéticos, produtividade e remoção de DQO}

Para facilitar a identificação das condições que promoveram o melhor desenvolvimento da Chlorella vulgaris, a tabela 1 reúne os parâmetros cinéticos (velocidades específicas de crescimento e tempos de geração), as produtividades de biomassa e as remoções da demanda química de oxigênio dos cultivos suplementados com diferentes concentrações de efluente de UASB.

Tabela 1: Parâmetros cinéticos, produtividades de biomassa e remoções de DQO dos cultivos de Chlorella vulgaris suplementados com diferentes concentrações de efluente de UASB.

\begin{tabular}{|c|c|c|c|c|}
\hline Efluente de UASB (\%) & $\begin{array}{c}\text { Velocidade específica } \boldsymbol{\mu}_{\text {máx }} \\
\left(\mathbf{h}^{-\mathbf{1}} \mathbf{)}\right.\end{array}$ & $\begin{array}{c}\text { Tempo de geração } \\
\mathbf{( h )}\end{array}$ & $\begin{array}{c}\text { Produtividade de biomassa } \\
\left(\mathbf{g} . \mathbf{L}^{-\mathbf{1}} \cdot \mathbf{d}^{-\mathbf{1}} \mathbf{)}\right.\end{array}$ & $\begin{array}{c}\text { Remoção de DQO } \\
\text { (\%) }\end{array}$ \\
\hline 20 & 0,0371 & 18,68 & 0,171 & 29,22 \\
\hline 30 & 0,0282 & 24,58 & 0,084 & 25,87 \\
\hline 50 & 0,0433 & 16,01 & 0,125 & 62,95 \\
\hline 70 & 0,0491 & 14,12 & 0,106 & 59,00 \\
\hline 80 & 0,0383 & 18,10 & 0,133 & 47,54 \\
\hline 100 & 0,0428 & 16,20 & 0,180 & 23,11 \\
\hline
\end{tabular}

O cultivo realizado com efluente de UASB na proporção de $70 \%$ apresentou a melhor velocidade específica de crescimento e, consequentemente, o melhor tempo de geração, indicando que essa concentração de efluente de UASB foi a que melhor promoveu o desenvolvimento da microalga. Os cultivos de $50 \%$ e $100 \%$ também apresentaram excelentes resultados de velocidade específica e de tempo de geração.

Antonio et al. (2013) cultivou a microalga Chlorella vulgaris em meio WC (Wright's Cryptophyte) modificando intensidade de luz, concentração celular inicial e concentração inicial de nitrato, em erlenmeyers de $500 \mathrm{~mL}$, contendo $300 \mathrm{~mL}$ de suspensão celular, a $25^{\circ} \mathrm{C}$, e obtiveram uma velocidade específica máxima de $0,0138 \mathrm{~h}^{-1}$. Melo (2015), cultivou a Chlorella vulgaris em meio BBM suplementado com vinhaça, obtendo velocidade específica máxima de $0,0200 \mathrm{~h}^{-1}$ e tempo de geração de $34,657 \mathrm{~h}$. Comparandose esses dados com os resultados apresentados na tabela 1, observa-se que o efluente de UASB promoveu uma melhor cinética de crescimento microalgal.

Analisando-se a produtividade dos cultivos, percebe-se que a Chlorella vulgaris alcançou maior valor $\left(0,180 \mathrm{~g} \cdot \mathrm{L}^{-1} \cdot \mathrm{d}^{-1}\right)$ em $100 \%$ de efluente de UASB. Porém, isso pode ter ocorrido pelo incremento dos sais do efluente à biomassa seca, mascarando o real valor da sua produtividade. Entre os demais cultivos destaca-se o de $20 \%$ de efluente de UASB, com uma produtividade em biomassa de $0,171 \mathrm{~g} \cdot \mathrm{L}^{-1} \cdot \mathrm{d}^{-1}$, cujo valor é justificado pela predominância do meio sintético específico da microalga, onde a mesma está adaptada e desenvolvese melhor.

Para melhorar a viabilidade econômica dos cultivos, podem-se destacar as proporções de 50\% e 80\%, com produtividades de 0,125 e $0,133 \mathrm{~g} \cdot \mathrm{L}^{-1} \cdot \mathrm{d}^{-1}$, respectivamente. Dentre esses, o cultivo de $50 \%$ mostrou-se superior em relação ao número final de células, velocidade específica de crescimento e tempo de geração. A tabela 1 também apresenta os valores de remoção de DQO dos cultivos de Chlorella vulgaris suplementados com efluente de UASB. Pode-se observar que nas concentrações de 50\% e 70\% de efluente de UASB a 
microalga reduziu a DQO em $62,95 \%$ e $59,00 \%$, sendo esses os melhores resultados e proporções para promover o tratamento do efluente.

Percebe-se que, ao se aumentar a concentração do efluente, a remoção de DQO diminui. Isso está associado ao aumento da carga orgânica refratária presente no efluente UASB, que é de difícil assimilação por microrganismos, uma vez que a maior parte da matéria biodegradável foi consumida durante o tratamento anaeróbio do esgoto sanitário.

Torres et al. (2014), ao cultivar Chlorella sp. em efluente de UASB autoclavado, obtiveram $36,8 \%$ de remoção de DQO como melhor resultado, valor menor que o alcançado neste estudo. Isso indica que a desinfecção prévia do efluente, além de não promover aumento na geração de lipídios, também não melhorou a remoção de DQO, podendo ser descartada para minimizar nos custos energéticos.

Silva (2014) utilizou esgoto sanitário biodigerido para cultivar a microalga Scenedesmus sp., em reatores tipo airlift, e atingiu uma remoção de DQO de 36\%, inferior a remoção alcançada no presente estudo com a Chlorella vulgaris. Com isso, pode-se destacar o potencial da espécie Chlorella vulgaris como auxílio no tratamento de efluentes domésticos.

Melo (2015), ao cultivar Chlorella vulgaris em meio BBM suplementado com vinhaça, conseguiu uma remoção de DQO de $87,57 \%$, reduzindo a DQO do meio de $1528 \mathrm{mg} \cdot \mathrm{L}^{-1}$ para $190 \mathrm{mg} \cdot \mathrm{L}^{-1}$, destacando a resistência dessa microalga e a sua eficácia no tratamento de efluentes agroindustriais. Os dados obtidos no presente estudo destacam a importância da Chlorella vulgaris como alternativa para o saneamento, por promoverem a biorremediação dos efluentes, gerando biomassa que pode ser utilizada como fonte de energia e favorecem a redução dos impactos gerados com o lançamento inadequado de efluentes, ricos em nutrientes, em corpos hídricos.

\section{Lipídios totais}

A partir da concentração ótima ( $50 \%$ de efluente de UASB), obtida por meio dos estudos realizados na seção anterior, foi realizado um cultivo em maior escala (fotobiorreator de $6 \mathrm{~L}$ ) para assim quantificar o teor de lipídios totais contido na biomassa microalgal. Conforme descrito anteriormente, foi empregada a metodologia descrita por Bligh et al. (1959) na extração dos lipídios e assim foi possível determinar que, para uma concentração de $50 \%$ de efluente de UASB, a Chlorella vulgaris apresentou um teor de lipídios totais de $18,14 \%$.

Torres et al. (2014) cultivou a Chlorella sp. em efluente de UASB autoclavado, com pH10,0 e DQO de $162,7 \mathrm{mg} . \mathrm{L}^{-1}$, obtendo um teor de lipídios de 13,2\%. Corroborando com o presente estudo, pode-se dizer que a desinfeç̧ão do efluente antes do cultivo não incrementou a produtividade lipídica. Pelo contrário, enfatizou a capacidade de crescimento e de geração de lipídios da Chlorella vulgaris em efluente não autoclavado, tornando essa espécie de microalga interessante para produção de biodiesel em larga escala, uma vez que ela dispensa custos com possíveis desinfecções do efluente. 
Matosi et al. (2015) cultivou a microalga Chlorella sp. utilizando meio BBM suplementado com concentrado de dessalinização em fotobiorreatores cônicos de 4,0L, onde as microalgas alcançaram um teor de lipídios entre 8,5-11,4\%. Já Melo (2015) avaliou a produção lipídica da Chlorella vulgaris em meio BBM modificado e em meio BBM modificado suplementado com vinhaça, obtendo teores de lipídios de 14,7\% e $11,5 \%$, respectivamente.

Desse modo, destaca-se a potencialidade do efluente de UASB para o cultivo de microalgas visando à produção de biodiesel, uma vez que o mesmo ofereceu condições favoráveis para que a Chlorella vulgaris alcançasse o teor de lipídios totais de 18,14\%, próximo aos 18,7\% apresentados por Rodolfi et al. (2009) para a respectiva microalga em condições ótimas de crescimento (cultivo realizado em meio sintético sem adição de efluentes).

\section{Análise Estatística}

Para verificar o nível de relação entre os parâmetros descritos na Tabela 1 foi realizado o estudo da matriz de correlação deles para cada microalga. A tabela 2 apresenta a Matriz de correlação entre os parâmetros avaliados para a Chlorella vulgaris. De maneira geral, observa-se que existem parâmetros que se correlacionam entre si, com correlações que chegam à ordem de $\pm 0,98$. Constata-se que a velocidade específica de crescimento é um fator importante que se relaciona com diversos parâmetros. Com o aumento dessa velocidade, ocorre uma redução no tempo de geração e um aumento na remoção de DQO.

A concentração de efluente de UASB apresentou correlação com a velocidade específica de crescimento e com o tempo de geração. Essas correlações indicam que um aumento nas concentrações de efluente de UASB acarreta aumento na velocidade e redução do tempo de geração. Não foram observadas correlações significativas da produtividade com os demais parâmetros. Uma maneira de identificar a formação de grupos com afinidades equivalentes é por meio da ACP. Nesse âmbito, a figura 4 apresenta os parâmetros que se relacionam e são semelhantes entre si.

Tabela 2: Matriz de correlação para a Chlorella vulgaris.

\begin{tabular}{|c|c|c|c|c|c|}
\hline Variáveis & Efluente de UASB & Velocidade específica & $\begin{array}{c}\text { Tempo de } \\
\text { geração }\end{array}$ & Remoção de DQo & Produtividade \\
\hline Efluente de UASB & 1 & & & & \\
\hline Velocidade específica & 0,55615654 & 1 & & & \\
\hline Tempo de geração & $-0,565270798$ & $-\mathbf{0 , 9 8 3 2 2 4 5 5}$ & 1 & & \\
\hline Remoção de DQO & 0,113207637 & $\mathbf{0 , 6 2 3 9 6 9 0 6 1}$ & $-0,59085917$ & 1 & 1 \\
\hline Produtividade & 0,28990958 & 0,249556685 & $-0,38727823$ & $-0,3728228$ & 1 \\
\hline
\end{tabular}

Observa-se que a projeção de variação dos dados nos dois eixos principais, explicam aproximadamente 85\% (Fator 1 + Fator 2) da variabilidade do processo, representando uma boa correlação entre os dados. Os resultados obtidos corroboram com a matriz de correlação para esta microalga, expondo a formação de elementos com dispersão semelhante. A ACP da figura 4 mostra a formação de 1 grupo correlacionado mais intimamente.

Esse grupo é composto pela concentração de efluente juntamente com a velocidade específica de crescimento, revelando que ocorre aumento na velocidade de crescimento à medida que aumenta a 
concentração de efluente no meio. Foram observadas correlações negativas, notando-se que à medida que a velocidade específica aumenta existe uma diminuição no tempo de geração, conforme já discutido na matriz de correlação. Destaca-se que a produtividade apresenta-se atuando de forma isolada dos demais parâmetros.

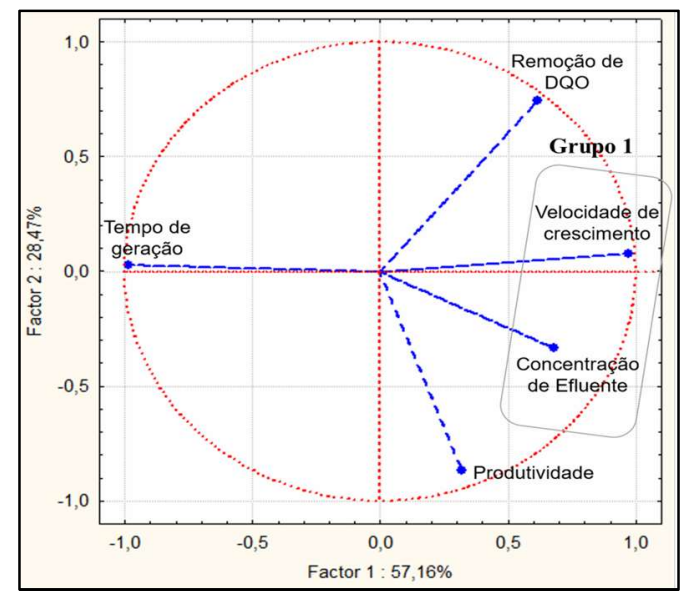

Figura 4: Análise em Componentes Principais para Chlorella vulgaris.

\section{CONCLUSÕES}

Perante o estudo do desenvolvimento da microalga Chlorella vulgaris por meio de suplementação nutricional realizada com efluente de tratamento anaeróbio de esgoto sanitário, direcionado à produção de biodiesel, pode-se concluir que a produtividade elevada da biomassa de microalgas a partir de águas residuárias, oriundas do tratamento de esgoto doméstico, sugere que este método de cultivo é viável para geração de biodiesel, avaliado como uma das muitas abordagens utilizadas para produção de energia sustentável e renovável.

Além disso, os cultivos suplementados com efluente de reator tipo UASB apresentaram excelente desenvolvimento celular, alcançando uma densidade máxima de $8,66 \times 10^{7}$ células. $\mathrm{mL}^{-1}$ em decorrência da adição de $50 \%$ do efluente. A Chlorella vulgaris no cultivo suplementado com $50 \%$ de efluente de UASB, além de ter alcançado o maior número de células, apresentou excelentes resultados nos demais parâmetros estudados: velocidade específica de crescimento, tempo de geração, produtividade e remoção de DQO, desenvolvendo um teor de lipídios de 18,14\%, próximo do valor obtido em condições ótimas, descrito na literatura, indicando que essa é a concentração de efluente mais recomendada para produção de biodiesel.

Todavia, as demais proporções de efluente de UASB também possibilitaram o desenvolvimento da microalga e podem ser utilizadas. A análise em componentes principais da microalga Chlorella vulgaris apresentou bastante afinidade entre o grupo formado pela concentração de efluente e a velocidade específica de crescimento.

\section{REFERÊNCIAS}

ANTONIO, P. R.; TEIXEIRA, C. M. L. L.; DE OLIVEIRA, M. C. R.. Avaliação das diferentes condições de cultivo da microalga Chlorella vulgaris visando o aumento da produtividade em biomassa. In: SIMPÓSIO NACIONAL DE BIOPROCESSOS, 19. Anais. Foz do Iguaçu: Hotel Bourbon Cataratas, 2013. 
BLIGH, E. G.; DYER, W. J.. A rapid method of total lipid extraction and purification. Canadian journal of biochemistry and physiology, v.37, n.8, p.911-917, 1959.

BRESSANI-RIBEIRO, T.; BRANDT, E. M. F.; GUTIERREZ, K. G.; DÍAZ, C. A.; GARCIA, G. B.; CHERNICHARO, C. A. L.. Potential of resource recovery in UASB/trickling filter systems treating domestic sewage in developing countries. Water Science and Technology, v.75, n.7, p.1659-1666, 2017.

CHERNiChARO, C. A. L.. Princípios do Tratamento Biológico de Águas Residuárias: reatores anaeróbios. 2 ed. Belo Horizonte: UFMG, 2007.

CHIRANJEEVI, P.; MOHAN, S. V.. Critical parametric influence on microalgae cultivation towards maximizing biomass growth with simultaneous lipid productivity. Renewable Energy, Amsterdã, v.98, p.64-71, 2016

CHISTI, Y.. Biodiesel from microalgae beats bioethanol. Trends in Biotechnology, Amsterdã, v.26, n.3, p.126-131, 2008.

DANTAS, A. O. G.. Influência do fotoperíodo no crescimento da Chlorella vulgaris (Chlorophyceae) visando produção de biodiesel. Biomolecular Engineering, Amsterdã, v.20, p.237242, 2010.

FERREIRA, S. P.; SOUZA-SOARES, L.; COSTA, J. A. V.. Revisão: microalgas: uma fonte alternativa na obtenção de ácidos gordos essenciais. Revista de Ciências Agrárias, Lisboa, v.36, n.3, p.275-287, 2013.

FRANCO, A. L. C.; LÔBO, I. P.; CRUZ, R. S.; TEIXEIRA, C. M. L. L.; NETO, J. A. D. A.; MENEZES, R. S.. Biodiesel de microalgas: avanços e desafios. Química Nova, São Paulo, v.36, n.3, p.437-448, 2013.

JUNIOR, S. D. O. N.; JUNIOR, S. N. M.; SEBASTIEN, N. Y.; FERRI, P.; COLDEBELLA, E. T.; AGUIAR, D. R.. Potencial de crescimento de Chlorella vulgaris em efluentes suínos para produção de biodiesel. Revista Brasileira de Energias Renováveis, Curitiba, v.2, p.23-33, 2013.

LIANG, Y.; SARKANY, N.; CUI, Y.. Biomass and lipid productivities of Chlorella vulgaris under autotrophic, heterotrophic and mixotrophic growth conditions. Biotechnology letters, Amsterdã, v.31, n.7, p.1043-1049, 2009.

MATOSI, A.; MORIOKAI, L. R. I.; SANT'ANNAI, E. S.; FRANÇAII, K. B.. Teores de proteínas e lipídeos de Chlorella sp. cultivada em concentrado de dessalinização residual. Ciência Rural, Santa Maria, v.45, n.2, p.364-370, 2015.

MELO, D. J. N.. Utilização de vinhaça no cultivo de Chlorella vulgaris. Dissertação (Mestrado em Engenharia Química) Universidade Federal de Campina Grande, Campina Grande, 2015.

MENEZES, R. S.; LELES, M. I. G.; SOARES, A. T.; FRANCO, P. I. B.; ANTONIOSI FILHO, N. R.; VIEIRA, A. A. H.. Avaliação da potencialidade de microalgas dulcícolas como fonte de matéria-prima graxa para a produção de biodiesel. Química Nova, São Paulo, v.36, n.1, p.10-15, 2013.

RICE, E. W.; BAIRD, R. B.; EATON, A. D.; CLESCERI, L. S. Standard methods for the examination of water and wastewater. 22 ed. Washington: APHA, 2012.

RODOLFI, I.; ZITTELLI, C. G.; BASSI, N.; PADOVANI, G.; BIONDI, N.; BIONDI, C.. Microalgae for oil: Strain selection, induction of lipid synthesis and outdoor mass cultivation in a low-cost photobioreactor. Biotechnology and bioengineering, Berlim, v.102, n.1, p.100-112, 2009.

SAFI, C.; ZEBIB, B.; MERAH, O.; PONTALIER, P. Y.; VACAGARCIA, C.. Morphology, composition, production, processing and applications of Chlorella vulgaris: a review. Renewable and Sustainable Energy Reviews, Amsterdã, v.35, p.265-278, 2014.

SCHMIDELL, W.; LIMA, A. U.; AQUARONE, E.; BORZANI, W.. Biotecnologia Industrial. 2 ed. São Paulo: Edgard Blücher, 2001.

SILVA, D. A.. Produção de biomassa de microalgas cultivadas em esgoto sanitário biodigerido visando a produção de biodiesel. Dissertação (Mestrado em Engenharia e Ciência dos Materiais) - Universidade Federal do Paraná, Curitiba, 2014.

TORRES, H. S. J.; CASSINI, S. T. A.; GONÇALVES, R. F.. Isolamento, sobrevivência e caracterização da biomassa de microalgas cultivadas em efluente de tratamento de esgoto sanitário visando a produção de biocombustíveis. In: SIMPÓSIO INTERNACIONAL DE QUALIDADE AMBIENTAL, 9. Anais. Porto Alegre: 2014.

VIEIRA, T. Q.; FERREIRA, W. B.; ARAÚJO, H. W. C.; CUNHA, T. H. C. S.; VIDAL, I. C. A.; MELO, D. J. N.. Estudo da viabilidade do uso de resíduos líquidos no cultivo da microalga Chlorella sp. visando a produção de biocombustíveis. Revista Monografias Ambientais, Santa Maria, v.13, n.4, p.34773490, 2014.

A CBPC - Companhia Brasileira de Produção Científica (CNPJ: 11.221.422/0001-03) detém os direitos materiais desta publicação. Os direitos referem-se à publicação do trabalho em qualquer parte do mundo, incluindo os direitos às renovações, expansões e disseminações da contribuição, bem como outros direitos subsidiários. Todos os trabalhos publicados eletronicamente poderão posteriormente ser publicados em coletâneas impressas sob coordenação da Sustenere Publishing, da Companhia Brasileira de Produção Científica e seus parceiros autorizados. Os (as) autores (as) preservam os direitos autorais, mas não têm permissão para a publicação da contribuição em outro meio, impresso ou digital, em português ou em tradução. 